\title{
Porphyrobacter mercurialis sp. nov., isolated from a stadium seat and emended description of the genus Porphyrobacter
}

David A Coil, Jennifer C Flanagan, Andrew Stump, Alexandra Alexiev, Jenna M Lang, Jonathan A Eisen

A novel, Gram-negative, non-spore-forming, pleomorphic yellow-orange bacterial strain was isolated from a stadium seat. Strain Coronado ${ }^{\top}$ falls within the Erythrobacteraceae family and the genus Porphyrobacter based on 16S rRNA phylogenetic analysis. This strain has Q-10 as the predominant respiratory lipoquinone, as do other members of the family. The fatty acid profile of this strain is similar to other Porphyrobacter, however Coronado ${ }^{\top}$ contains predominately $\mathrm{C} 18: 1 \omega 7 \mathrm{cis}$ and $\mathrm{C} 16: 0$, a high percentage of the latter not being observed in any other Erythrobacteraceae. This strain is catalase-positive and oxidasenegative, can grow from $4-28{ }^{\circ} \mathrm{C}$, at $\mathrm{NaCl}$ concentrations $0.1-1.5 \%$, and at $\mathrm{pH} 6.0-8.0$. On the basis of phenotypic and phylogenetic data presented in this study, strain Coronado ${ }^{\top}$ represents a novel species in the Porphyrobacter genus for which the name Porphyrobacter mercurialis sp. nov. is proposed; the type strain is Coronado ${ }^{\top}$ (=DSMZ 29971, =LMG 28700). 
1. Porphyrobacter mercurialis sp. nov., isolated

. from a stadium seat and emended description of the genus Porphyrobacter

David A. Coil ${ }^{\mathbf{1}}$, Jennifer C. Flanagan ${ }^{\mathbf{1}}$, Andrew Stump ${ }^{\mathbf{1}}$, Alexandra Alexiev ${ }^{\mathbf{1}}$, Jenna M. Lang ${ }^{\mathbf{1}}$, Jonathan A. Eisen ${ }^{\mathbf{1 , 2}, \#,}$ 1 University of California Davis Genome Center, Davis, CA, USA. 2 University of California Davis Department of Evolution and Ecology, Department of Medical Microbiology and Immunology, Davis, California, USA \# Corresponding author: jaeisen@ucdavis.edu

October 21, 2015

\begin{abstract}
Abstract

A novel, Gram-negative, non-spore-forming, pleomorphic yellow-orange bacterial strain was isolated from a stadium seat. Strain CoronadoT falls within the Erythrobacteraceae family and the genus Porphyrobacter based on 16S rRNA phylogenetic analysis. This strain has Q-10 as the predominant respiratory lipoquinone, as do other members of the family. The fatty acid profile of this strain is similar to other Porphyrobacter, however

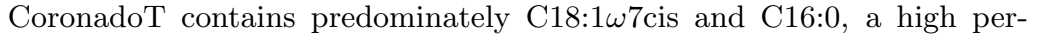
centage of the latter not being observed in any other Erythrobacteraceae. This strain is catalase-positive and oxidase-negative, can grow from 4-28 ${ }^{\circ} \mathrm{C}$, at $\mathrm{NaCl}$ concentrations $0.1-1.5 \%$, and at $\mathrm{pH}$ 6.0-8.0. On the basis of phenotypic and phylogenetic data presented in this study, strain CoronadoT represents a novel species in the Porphyrobacter genus for which the name Porphyrobacter mercurialis sp. nov. is proposed; the type strain is CoronadoT (=DSMZ 29971, =LMG 28700).
\end{abstract}

\title{
INTRODUCTION
}

In this study, strain CoronadoT was isolated from a stadium seat at Niedermeyer Field, Coronado High School in Coronado, California, USA as part of a nationwide Citizen Science project (Project MERCCURI - www.spacemicrobes.org.) One goal of Project MERCCURI was to collect bacterial isolates to be used for an experiment aboard the International Space Station (ISS). The 16S rRNA gene sequenced from this particular isolate was at least $99 \%$ identical to rRNA 
genes from a few uncultured organisms. Uncultured isolates with high identity $(=>99 \%)$ were found in samples from deep ocean sediment and the human skin microbiome. However, the highest identity to a cultured organism (Porphyrobacter donghaensis) [28] was only $95.5 \%$ (as determined by BLAST, [1]). Given the low identity to characterized species, a more detailed study of this isolate was undertaken.

A phylogenetic analysis of the Alphaproteobacteria class led in 2005 to the creation of a new family, Erythrobacteraceae, to house the genera Erythrobacter, Porphyrobacter and Erythromicrobium [16]. These genera were later joined by Altererythrobacter [15] and Croceicoccus [25], the latter work also emended the description of the family. Members of the Erythrobacteraceae family are Gram-negative, aerobic bacteria that contain carotenoids, usually appearing pink, orange or yellow. They do not form spores, are chemo-organotrophic, and are most often associated with aquatic environments. The Porphyrobacter genus was established in 1993 with the description of Porphyrobacter neustonensis, isolated from freshwater [5]. All subsequent Porphyrobacter species have also been isolated from aquatic sources (hot springs, seawater, and swimming pools) including P. tepidarius [8], P. sanguineus [9], P. cryptus [19], P. donghaensis [28], P. dokdonensis [27] and P. colymbi [6].

Phylogenetic and biochemical characteristics presented here show that our isolate is clearly distinct from other members within the Erythrobacteraceae family and is most closely related to the genus Porphyrobacter. However, a major taxonomic revision of this family is most likely required, as has been suggested by others (e.g. [19] [11]). Here we describe the genotypic, morphologic, and biochemical characteristics of strain CoronadoT, based on which we propose the name of Porphyrobacter mercurialis sp. nov.

\section{METHODS}

Cells were initially grown on plates containing either Reasoner's $2 \mathrm{~A}$ agar (R2A), or lysogeny broth agar (LB). LB was made with $10 \mathrm{~g}$ tryptone, $10 \mathrm{~g}$ $\mathrm{NaCl}$, and $5 \mathrm{~g}$ yeast extract per liter. A clear preference for growth on LB was observed and so was used for all subsequent experiments. Salt tolerance was measured by growth in liquid media $\left(25^{\circ} \mathrm{C}\right)$ from $0 \%$ to $20 \% \mathrm{w} / \mathrm{v} \mathrm{NaCl}$. pH tolerance was measured by growth in liquid media $\left(25^{\circ} \mathrm{C}\right)$ from $\mathrm{pH} 3.4$ to $\mathrm{pH}$ 8.0. Temperature preference was measured by growth in liquid culture across the range $4{ }^{\circ} \mathrm{C}$ to $30^{\circ} \mathrm{C}$. Growth in microgravity (OD600) was measured aboard the International Space Station (ISS).

Cell morphology, motility, and presence/absence of flagella were examined by light microscopy (Zeiss Axio Lab.A1) and transmission electron microscopy (TEM). Cell cultures in either exponential or stationary phase were prepared for TEM by the UC Davis Electron Microscopy lab as follows. 400 mesh copper grids with formvar/carbon support film (Ted Pella, Inc., Redding, Ca.) were placed on dental wax. A $10 \mu \mathrm{l}$ drop of fixed or unfixed sample was placed onto the grid and left in a dust-free environment for 10 minutes. Then excess was wicked off with filter paper. A $10 \mu \mathrm{l}$ drop of $1 \%$ PTA pH 5.8 (phosphotungstic acid) or $1 \%$ ammonium molybdate in double-distilled water was added to the grid and wicked off immediately. Grids were allowed to air-dry completely 
before viewing in a Philips CM120 (FEI/Philips Inc, Hillsborough, Or.) electron microscope at $80 \mathrm{KV}$.

Oxidase activity was measured using a solution of tetramethyl-p-phenylenediamine and catalase activity was measured by the addition of hydrogen peroxide to plated cells. The hydrolysis of starch and casein were measured by standard plate methods (beef agar with soluble starch and iodine staining, and milk agar with a pancreatic digest of casein respectively). Carbon source oxidation was assayed using the Phenotypic MicroArray (TM) services offered by Biolog, Inc. using their standard procedures for gram-negative bacteria as follows. Colonies were grown on blood agar at room temperature and suspended in IF-0a inoculating fluid (Biolog) to a density of $42 \%$ transmittance. The cell suspension was diluted 1:6 in IF-0a plus 1x Dye $\mathrm{H}$ (Biolog) and a carbon source utilization MicroPlate (PM1; Biolog) was inoculated with $100 \mu \mathrm{l}$ per well. The PM1 microplate was incubated at $23{ }^{\circ} \mathrm{C}$ and read by the OmniLog instrument every 15 minutes for 96 hours. Duplicate sets of OmniLog data were converted to average read value and a threshold of 78 was required in both replicates for a positive call.

Respiratory quinones, polar lips, and fatty acids

Cells were grown to post exponential phase ( $\sim 72$ hours) in $1 \mathrm{~L}$ of $\mathrm{LB}$ at $23{ }^{\circ} \mathrm{C}$ for large-scale biomass production, then centrifuged to pellet cells and lyopholized (VirTis Freezemobile). Analysis of respiratory quinones/polar lipids and fatty acids were carried out by the Identification Service, DSMZ, Braunschweig. Germany. Protocol details and references can be found at the following DSMZ pages; https://www.dsmz.de/services/services-microorganisms/ identification/analysis-of-polar-lipids.html, https://www.dsmz.de/ services/services-microorganisms/identification/analysis-of-respiratory-quinones. html, https://www.dsmz.de/services/services-microorganisms/identification/ analysis-of-cellular-fatty-acids.html.

$16 \mathrm{~S}$ rDNA, genome sequencing, and phylogenetic analysis

Genomic DNA was extracted using a Wizard Genomic DNA Purification Kit (Promega). A nearly full-length 16S rRNA gene sequence was amplified using the 27F (5'-AGAGTTTGATCMTGGCTCAG-3') and 1391R (5'-GACGGGCGGTGTGTRCA3') "universal" primers. Sanger sequencing was provided by the College of Biological Science UC-DNA Sequencing Facility (UC Davis). This DNA was also used for Illumina sequencing of the draft genome as described elsewhere (Coil and Eisen, in press). The genome sequence was annotated using the RAST server [2] [18].

The 1482bp 16S rDNA sequence was obtained from the genome assembly in RAST (GenBank: KP122961), and uploaded to the Ribosomal Database Project (RDP) [3]. Since the RDP database is incomplete with respect to the Erythrobacteraceae family, additional type strain sequences were obtained from NCBI to ensure that every member of the family with official standing in nomenclature (http://www.bacterio.net/) was present in the alignment downloaded from RDP. Because the taxon names exported with this alignment contained special characters that were not compatible with phylogenetic reconstruction software, a custom script was used to remove or replace 
those characters with underscores. A description of and link to this script can be found in [4]. The alignment was manually examined using MView (http://www.ebi.ac.uk/Tools/msa/mview/t), the secondary structure was generated using the RNAfold Web Server (http://rna.tbi.univie.ac.at/cgi-bin/ RNAfold.cgi) and visualized with Forna (http://nibiru.tbi.univie.ac.at/ forna/). This alignment was used to generate phylogenetic trees using a variety of methods including maximum likelihood (RAxML, [22]), Bayesian (MrBayes, [20] [12]), neighbor-joining (MEGA6, [23], NINJA, http://nimbletwist.com/ software/ninja/), and maximum parsimony (MEGA6). Dendroscope 3 [13] and FigTree (http://tree.bio.ed.ac.uk/software/figtree/) were used to view and edit the phylogenetic trees.

\section{RESULTS AND DISCUSSION}

Morphological, physiological, and biochemical characteristics

Cells were non-motile and not observed to form spores or possess flagella, though over 30 flagella or flagella-associated genes are present in the genome. In contrast, the genome for a non-motile close relative (Porphyrobacter cryptus, GenBank: ASM42298v1) does not contain any flagellar genes so it is possible that strain CoronadoT is motile under specific conditions. Unlike most members of the Erythrobacteraceae family, strain CoronadoT is oxidase-negative. This strain is catalase-positive, and unable to hydrolze casein or starch.

Cells were oval or rod shaped and ranged in length from $1.2 \mu \mathrm{m}$ to $2.2 \mu \mathrm{m}$ with an average of $1.6 \mu \mathrm{m}$ (Figure 1). Cell width ranged from $0.6 \mu \mathrm{m}$ to $1.0 \mu \mathrm{m}$ with an average of $0.8 \mu \mathrm{m}$.

Growth was only observed under aerobic conditions, from $4{ }^{\circ} \mathrm{C}$ to $28^{\circ} \mathrm{C}$, with optimal growth around $25{ }^{\circ} \mathrm{C}$. No growth was observed under microaerophilic conditions (culture caps closed). Low levels of growth were observed at $\mathrm{pH} 6.0$ up to $\mathrm{pH} 8.0$, maximum growth occurred around neutral $\mathrm{pH}$. $\mathrm{NaCl}$ was required for growth, and the strain could not grow at $>1.5 \% \mathrm{NaCl}$, optimal growth was at $0.5 \% \mathrm{NaCl}$. No statistically significant difference in growth was observed between earth and microgravity aboard the International Space Station (ISS).

Strain CoronadoT could oxidize the following as sole carbon sources: GlycylL-Glutamic Acid, L-Rhamnose, D-Mannose, D-Trehalose, a-D-Glucose, L-Fucose, D-Galactose, Citric acid, D-Glucuronic acid, D-Galactonic acid, L-Galactonic acid- $\gamma$-Lactone, Acetoacetic acid, Acetic acid, Pyruvic acid, and L-Malic acid.

The strain was unable to grow on N-Acetyl-D-Glucosamine, D-Saccharic Acid, Succinic Acid, L-Aspartic Acid, L-Proline, D-Alanine, Dulcitol, D-Serine, D-Sorbitol, Glycerol, D-Gluconic Acid, D,L- $\alpha$-Glycerol-Phosphate, L-Lactic Acid, Formic Acid, D-Mannitol, L-Glutamic Acid, D-Glucose-6-Phosphate, D-Galactonic Acid- $\gamma$-Lactone, D,L-Malic Acid, Tween 20, D-Fructose, Maltose, D-Melibiose, Thymidine, L-Asparagine, D-Aspartic Acid, D-Glucosaminic Acid, 1,2-Propanediol, Tween 40, $\alpha$-Keto-Glutaric Acid, $\alpha$-Keto-Butyric Acid, $\alpha$-Methyl-D-Galactoside, $\alpha$-D-Lactose, Lactulose, Sucrose, Uridine, L-Glutamine, m-Tartaric Acid, DGlucose-1-Phosphate, D-Fructose-6-Phosphate, Tween 80, $\alpha$-Hydroxy Glutaric Acid- $\gamma$-Lactone, $\alpha$-Hydroxy Butyric Acid, $\beta$-Methyl-D-Glucoside, Adonitol, Maltotriose, 2-Deoxy Adenosine, Adenosine, Glycyl-L-Aspartic Acid, m-Inositol, DThreonine, Fumaric Acid, Bromo Succinic Acid, Propionic Acid, Mucic Acid, 
Glycolic Acid, Glyoxylic Acid, D-Cellobiose, Inosine, Tricarballylic Acid, LSerine, L-Threonine, L-Alanine, L-Alanyl-Glycine, Acetoacetic Acid, N-Acetyl$\beta$-D-Mannosamine, Mono Methyl Succinate, Methyl Pyruvate, D-Malic Acid, Glycyl-L-Proline, p-Hydroxy Phenyl Acetic Acid, m-Hydroxy Phenyl Acetic Acid, Tyramine, D-Psicose, Glucuronamide, Phenylethyl-amine, or 2-Aminoethane.

Phylogeny and Genome analysis

Phylogenetic analysis was performed using the full length (1482 bp) 16S rDNA sequence from the genome assembly, not the shorter (1350 bp) version from Sanger sequencing. The CoronadoT $16 \mathrm{~S}$ rDNA sequence showed less than 95.5\% identity to other Porphyrobacter species and identity is even lower to other genera in the family. Given the low $16 \mathrm{~S}$ rDNA identity to other members of the family, we did not perform DNA-DNA hybridization as this would have been uninformative [21] [24] [17].

Phylogenetic trees built by varying the alignment and tree-building algorithms, number of taxa included, and choice of outgroup demonstrated both that the current taxonomy of the family is in need of revision (as has been suggested by others, e.g., [19] and [11]) and that the placement of CoronadoT within the family is not stable. Because the Bayesian and Maximum Likelihood methods show a very similar topology, and are considered the most accurate methods for phylogenetic analysis (e.g. [26], [14], [7]), we have shown those trees in Figure 2 and Figure 3. Both of these trees place CoronadoT within the Porphyrobacter clade, though in the Bayesian tree there is a polytomy at the base of this clade. We note, as also shown recently by [11], that this clade is always polyphyletic with respect to Erythromicrobium ramosum and often to Erythrobacter litoralis. In addition, CoronadoT is found on a long branch due to several changes that are unique to this strain, relative to the rest of the family. These changes are identical in both the assembly and the Sanger sequence and are all compatible with the secondary structure model of $16 \mathrm{~S}$ (e.g., changes in a stem nucleotide pair with the appropriate base). Based on this analysis, we chose to compare CoronadoT to the five other Porphyrobacter species listed in Table 1.

Analysis of the draft genome of strain CoronadoT was used to complement the physical characterizations typical of the family Erythrobacteraceae and the genus Porphyrobacter. For example, CoronadoT does not contain any of the numerous genes involved in bacteriochlorophyll biosynthesis, rendering protein extraction/spectrophotometry unnecessary. Conversely, while no flagella were observed by TEM, this strain appears to possess the required genes making it likely that the flagella were lost in sample preparation or that their expression is condition-dependent.

Polar lipid, respitory lipoquinone, and fatty acid methyl esters

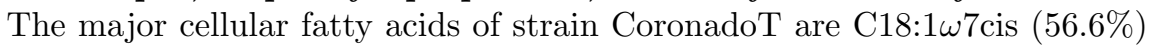
and C16:0 (20.3\%). Other fatty acids found in significant amounts $(>1 \%)$ are

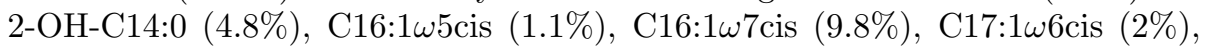
$\mathrm{C} 18: 1 \omega 5 \mathrm{cis}(1.1 \%)$, and C18:0 (1.2\%). The fatty acid profile of strain CoronadoT fits generally within the ranges described for members of the most closely related genera (Erythrobacter, Porphyrobacter and Erythromicrobium, comparison data 
from [10]). The two exceptions to this are a slightly higher level of C14:0 than average and a much higher level of C16:0 than average. However, it is difficult to compare across studies since variation in growth conditions can significantly influence the fatty acid profile.

The major respiratory quinone is ubiquinone $10(92 \%)$, as it is for all members of the Erythrobacteraceae family. The predominant polar lipid is phosphatidylglycerol, with significant amounts of sphingoglycolipid and phosphatidylethanolamine. Smaller amounts of diphosphatidylglycerol, phosphatidylcholine, and two unidentified phospholipids were also observed (Figure 4).

\section{Conclusions}

Strain CoronadoT clearly falls within the Erythrobacteraceae family, based on both phylogenetic analysis and chemotaxonomic and molecular characteristics (notably fatty acid profile, carotenoid production, major respiratory quinone, and GC content). Using Bayesian and Maximum Likelihood phylogenetic reconstruction, the strain falls within a well-supported (but polyphyletic) Porphyrobacter clade. In addition, CoronadoT shares a number of characteristics with Porphyrobacter, including the fatty acid profile, polar lipid composition, catalase activity, etc. The largest differences are the tolerance for growth at lower temperatures, elevated C16:0, and the lack of bacteriochlorophyll $a$ (for the latter of which we have proposed emending the genus description). These characteristics, in combination with the phylogenetic analysis, lead us to propose that CoronadoT be classified as Porphyrobacter mercurialis sp. nov. In the future, the taxonomic status of this strain may change depending upon availability of clear and distinctive evidence for a new genus as per polyphasic taxonomic and/or genome sequence based taxonomic approaches.

\subsection{Emended description of the genus Porphyrobacter Fuerst et al 1993}

The description is identical to that given by [5] with the following amendments. Most species synthesize $\mathrm{BChl} a$ on low-nutrient media under aerobic and semiaerobic conditions. DNA base composition is 63.8 to $67.3 \mathrm{G}+\mathrm{C}$.

\subsection{Description of Porphyrobacter mercurialis sp. nov.}

Porphyrobacter mercurialis (mer.cur.ia.al'is L. adj. mercurialis, temperamental in reference to difficulties in establishing consistent growth requirements during characterization).

Gram-negative, non-spore forming pleomorphic bacteria. Strictly aerobic and chemoheterotrophic. Contains carotenoids, but not bacteriochlorophyll $a$. The major respiratory quinone is ubiquinone 10, the dominant phospholipds are phosphatidylglycerol, sphingoglycolipid, and phophatidylthanolamine. Colonies on LB are round, glossy, and less than $1 \mathrm{~mm}$ in diameter. Coloration on agar and in dense liquid culture is dark yellowish orange, appears lighter yellow in liquid culture during exponential phase. Morphology is non-motile pleomorphic ovals or short rods (average $1.6 \mu \mathrm{m}$ length), often with pointed ends. Growth in 
liquid culture from $4-28{ }^{\circ} \mathrm{C}$, optimum growth at $25{ }^{\circ} \mathrm{C}$. Requires salt for growth, optimal growth at $0.5 \% \mathrm{NaCl}$, cannot grow at $3 \% \mathrm{NaCl}$. Tolerates up to $\mathrm{pH}$ 8.0, optimum around neutral $\mathrm{pH}$. Oxidase-negative and catalase-positive, does not hydrolyze casein or starch. Does not grow on R2A agar. The primary fatty acids are $\mathrm{C} 18: 1 \omega 7$ cis and C16:0, with a high percentage of the latter relative to other species in the Erythrobacteraceae family. Can oxidize the following as sole carbon sources: Glycyl-L-Glutamic Acid, L-Rhamnose, D-Mannose, DTrehalose, a-D-Glucose, L-Fucose, D-Galactose, Citric acid, D-Glucuronic acid, D-Galactonic acid, L-Galactonic acid- $\gamma$-Lactone, Acetoacetic acid, Acetic acid, Pyruvic acid, and L-Malic acid.

The type strain CoronadoT (=DSMZ 29971, =LMG 28700) was isolated from a stadium seat in Coronado, California, USA. The GC content of the type strain is $67.3 \%$, as determined by genome sequencing. The genome size is approximately 3.5 MB.

\section{Acknowledgements}

The authors would like to thank the Coronado Pop Warner Islanders for initial collection of the sample and participation in Project MERCCURI, as well as Kris Tracy who assisted in the etymology of the proposed species name.

\section{$0.3 \quad$ Table 1}

Caption for Table 1 (Placed in document so that the appropriate references appear in the Bibliography, please remove in actual paper)

Phenotypic comparison of CoronadoT and other members of the Porphyrobacter genus. Positive $=+$, negative $=-$, No data available $=$ ND. Data from this study and [9], [19], [8], [28], [5], [27].

\section{References}

[1] SF Altschul, W Gish, W Miller, EW Myers, and DJ Lipman. Basic local alignment search tool. J Mol Biol, 215:403-10, Oct 1990.

[2] Ramy K Aziz, Daniela Bartels, Aaron A Best, Matthew DeJongh, Terrence Disz, Robert A Edwards, Kevin Formsma, Svetlana Gerdes, Elizabeth M Glass, Michael Kubal, Folker Meyer, Gary J Olsen, Robert Olson, Andrei L Osterman, Ross A Overbeek, Leslie K McNeil, Daniel Paarmann, Tobias Paczian, Bruce Parrello, Gordon D Pusch, Claudia Reich, Rick Stevens, Olga Vassieva, Veronika Vonstein, Andreas Wilke, and Olga Zagnitko. The RAST Server: Rapid Annotations using Subsystems Technology. BMC Genomics, 9(1):75, 2008.

[3] J. R. Cole, Q. Wang, J. A. Fish, B. Chai, D. M. McGarrell, Y. Sun, C. T. Brown, A. Porras-Alfaro, C. R. Kuske, and J. M. Tiedje. Ribosomal Database Project: data and tools for high throughput rRNA analysis. Nucleic Acids Research, 42(D1):D633-D642, nov 2013. 
[4] MI Dunitz, JM Lang, G Jospin, AE Darling, JA Eisen, and DA Coil. Swabs to genomes: a comprehensive workflow. PeerJ, 3:e960, null 2015.

[5] JA Fuerst, JA Hawkins, A Holmes, LI Sly, CJ Moore, and E Stackebrandt. Porphyrobacter neustonensis gen. nov., sp. nov., an aerobic bacteriochlorophyll-synthesizing budding bacterium from fresh water. Int J Syst Bacteriol, 43:125-34, Jan 1993.

[6] K Furuhata, A Edagawa, H Miyamoto, Y Kawakami, and M Fukuyama. Porphyrobacter colymbi sp. nov. isolated from swimming pool water in Tokyo, Japan. J Gen Appl Microbiol, 59:245-50, 2013.

[7] BG Hall. Comparison of the accuracies of several phylogenetic methods using protein and DNA sequences. Mol Biol Evol, 22:792-802, Mar 2005.

[8] S Hanada, Y Kawase, A Hiraishi, S Takaichi, K Matsuura, K Shimada, and KV Nagashima. Porphyrobacter tepidarius sp. nov., a moderately thermophilic aerobic photosynthetic bacterium isolated from a hot spring. Int J Syst Bacteriol, 47:408-13, Apr 1997.

[9] A Hiraishi, Y Yonemitsu, M Matsushita, YK Shin, H Kuraishi, and K Kawahara. Characterization of Porphyrobacter sanguineus sp. nov., an aerobic bacteriochlorophyll-containing bacterium capable of degrading biphenyl and dibenzofuran. Arch Microbiol, 178:45-52, Jul 2002.

[10] Akira Hiraishi, Yoshitomo Yonemitsu, Mayumi Matsushita, Yong Shin, Hiroshi Kuraishi, and Kazuyoshi Kawahara. Characterization of Porphyrobacter sanguineus sp. nov. an aerobic bacteriochlorophyll-containing bacterium capable of degrading biphenyl and dibenzofuran. Archives of $\mathrm{Mi}$ crobiology, 178(1):45-52, jul 2002.

[11] Y Huang, Y Zeng, H Feng, Y Wu, and X Xu. Croceicoccus naphthovorans sp. nov., a polycyclic aromatic hydrocarbons-degrading and acylhomoserine-lactone-producing bacterium isolated from marine biofilm, and emended description of the genus Croceicoccus. Int J Syst Evol Microbiol, 65:1531-6, May 2015.

[12] JP Huelsenbeck and F Ronquist. MRBAYES: Bayesian inference of phylogenetic trees. Bioinformatics, 17:754-5, Aug 2001.

[13] DH Huson and C Scornavacca. Dendroscope 3: an interactive tool for rooted phylogenetic trees and networks. Syst Biol, 61:1061-7, 2012.

[14] MK Kuhner and J Felsenstein. A simulation comparison of phylogeny algorithms under equal and unequal evolutionary rates. Mol Biol Evol, 11:459-68, May 1994.

[15] KK Kwon, JH Woo, SH Yang, JH Kang, SG Kang, SJ Kim, T Sato, and C Kato. Altererythrobacter epoxidivorans gen. nov., sp. nov., an epoxide 
hydrolase-active, mesophilic marine bacterium isolated from cold-seep sediment, and reclassification of Erythrobacter luteolus Yoon et al. 2005 as Altererythrobacter luteolus comb. nov. Int J Syst Evol Microbiol, 57:220711, Oct 2007.

[16] KB Lee, CT Liu, Y Anzai, H Kim, T Aono, and H Oyaizu. The hierarchical system of the 'Alphaproteobacteria': description of Hyphomonadaceae fam. nov., Xanthobacteraceae fam. nov. and Erythrobacteraceae fam. nov. Int J Syst Evol Microbiol, 55:1907-19, Sep 2005.

[17] JP Meier-Kolthoff, M Göker, C Spröer, and HP Klenk. When should a DDH experiment be mandatory in microbial taxonomy? Arch Microbiol, 195:413-8, Jun 2013.

[18] R. Overbeek, R. Olson, G. D. Pusch, G. J. Olsen, J. J. Davis, T. Disz, R. A. Edwards, S. Gerdes, B. Parrello, M. Shukla, V. Vonstein, A. R. Wattam, F. Xia, and R. Stevens. The SEED and the Rapid Annotation of microbial genomes using Subsystems Technology (RAST). Nucleic Acids Research, 42(D1):D206-D214, nov 2013.

[19] FA Rainey, J Silva, MF Nobre, MT Silva, and Costa MS da. Porphyrobacter cryptus sp. nov., a novel slightly thermophilic, aerobic, bacteriochlorophyll a-containing species. Int J Syst Evol Microbiol, 53:35-41, Jan 2003.

[20] F Ronquist and JP Huelsenbeck. MrBayes 3: Bayesian phylogenetic inference under mixed models. Bioinformatics, 19:1572-4, Aug 2003.

[21] E. Stackebrandt and B. M. Goebel. Taxonomic Note: A Place for DNADNA Reassociation and 16S rRNA Sequence Analysis in the Present Species Definition in Bacteriology. International Journal of Systematic Bacteriology, 44(4):846-849, oct 1994.

[22] A Stamatakis. RAxML version 8: a tool for phylogenetic analysis and post-analysis of large phylogenies. Bioinformatics, 30:1312-3, May 2014.

[23] K Tamura, G Stecher, D Peterson, A Filipski, and S Kumar. MEGA6: Molecular Evolutionary Genetics Analysis version 6.0. Mol Biol Evol, 30:2725-9, Dec 2013.

[24] BJ Tindall, R Rosselló-Móra, HJ Busse, W Ludwig, and P Kämpfer. Notes on the characterization of prokaryote strains for taxonomic purposes. Int J Syst Evol Microbiol, 60:249-66, Jan 2010.

[25] XW Xu, YH Wu, CS Wang, XG Wang, A Oren, and M Wu. Croceicoccus marinus gen. nov., sp. nov., a yellow-pigmented bacterium from deep-sea sediment, and emended description of the family Erythrobacteraceae. Int J Syst Evol Microbiol, 59:2247-53, Sep 2009.

[26] Z Yang and B Rannala. Molecular phylogenetics: principles and practice. Nat Rev Genet, 13:303-14, Mar 2012. 
[27] JH Yoon, SJ Kang, MH Lee, HW Oh, and TK Oh. Porphyrobacter dokdonensis sp. nov., isolated from sea water. Int J Syst Evol Microbiol, 56:107983, May 2006.

[28] JH Yoon, MH Lee, and TK Oh. Porphyrobacter donghaensis sp. nov., isolated from sea water of the East Sea in Korea. Int J Syst Evol Microbiol, 54:2231-5, Nov 2004. 
1

Figure 1: Transmission Electron Microscopy (TEM)

Transmission Electron Microscopy (TEM) of exponential phase culture of Coronado ${ }^{\top}$ in lysogeny broth (LB), grown at $23^{\circ} \mathrm{C}$. Cells were negatively stained with Ammonium Molybdate.

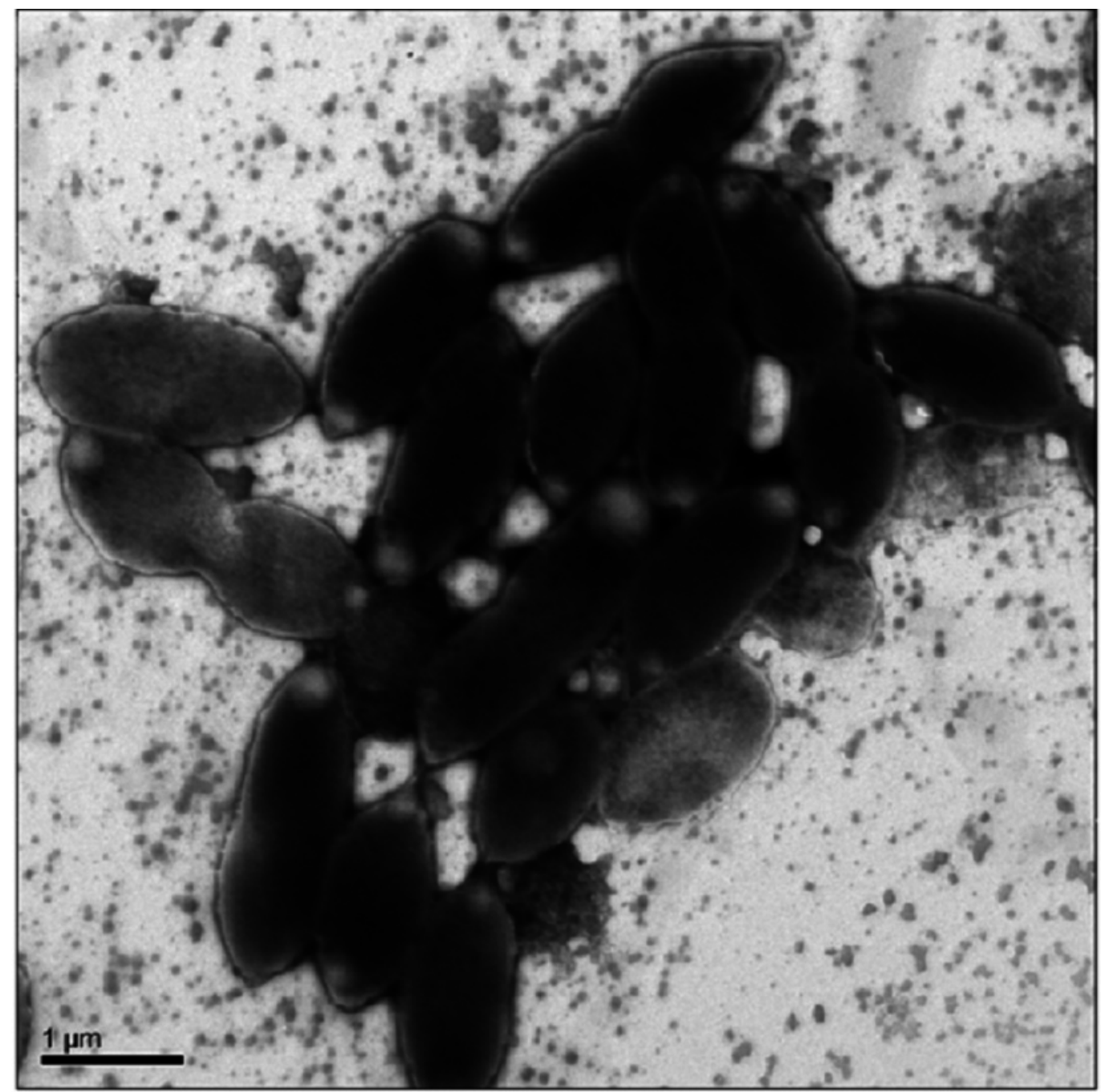




\section{Figure 2 (on next page)}

Figure 2: Maximum Likelihood tree

Figure 2: Maximum Likelihood tree based on the 16S rRNA gene sequence of Coronado ${ }^{\top}$ and all type strains from the Erythrobacteraceae family. Tree was inferred from an Infernal-based alignment created in RDP using RAxML with the gamma model of rate heterogeneity. Numbers at the nodes (only values $>70$ are shown) represent support values for 1000 bootstrap replicates. The tree was rooted to Novosphingobium indicum as an outgroup since this species was shown to be one of the closest relatives to the Erythrobacteraceae family in a tree of all Alphaproteobacteria. 


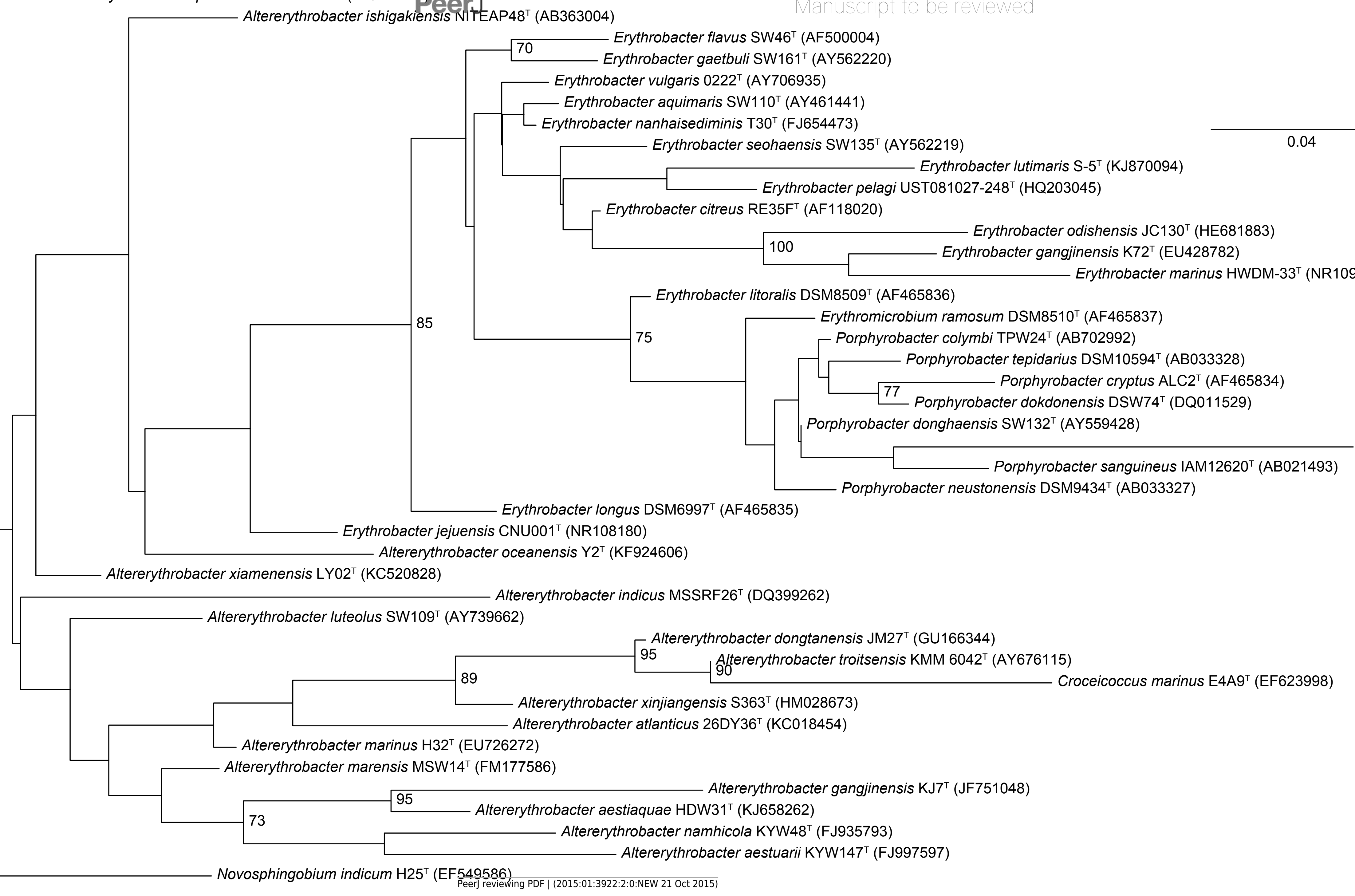




\section{Figure 3 (on next page)}

Figure 3: Bayesian tree

Figure 3: Bayesian tree based on the same sequence alignment described in Figure 2 (Infernal-based RDP alignment of 16S rRNA gene sequence of Coronado ${ }^{\top}$ and all type strains from the Erythrobacteraceae family). Tree was inferred using MrBayes with the General Time Reversible (GTR) substitution model. Numbers at nodes (only values $>70$ are shown) represent posterior probability values from 1,000,000 iterations of the tree, with a $25 \%$ relative burnin. The tree was rooted to Novosphingobium indicum as an outgroup since this species was shown to be one of the closest relatives to the Erythrobacteraceae family in a tree of all Alphaproteobacteria. 


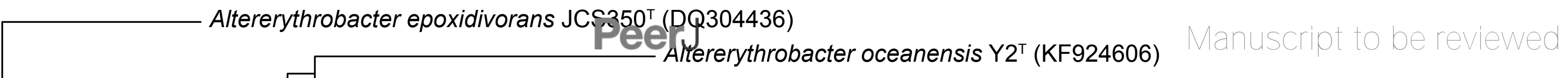

Erythrobacter jejuensis $\mathrm{CNU}^{001^{\top}}$ (NR108180)

Altererythrobacter ishigakiensis NITEAP48 ${ }^{\top}$ (AB363004)

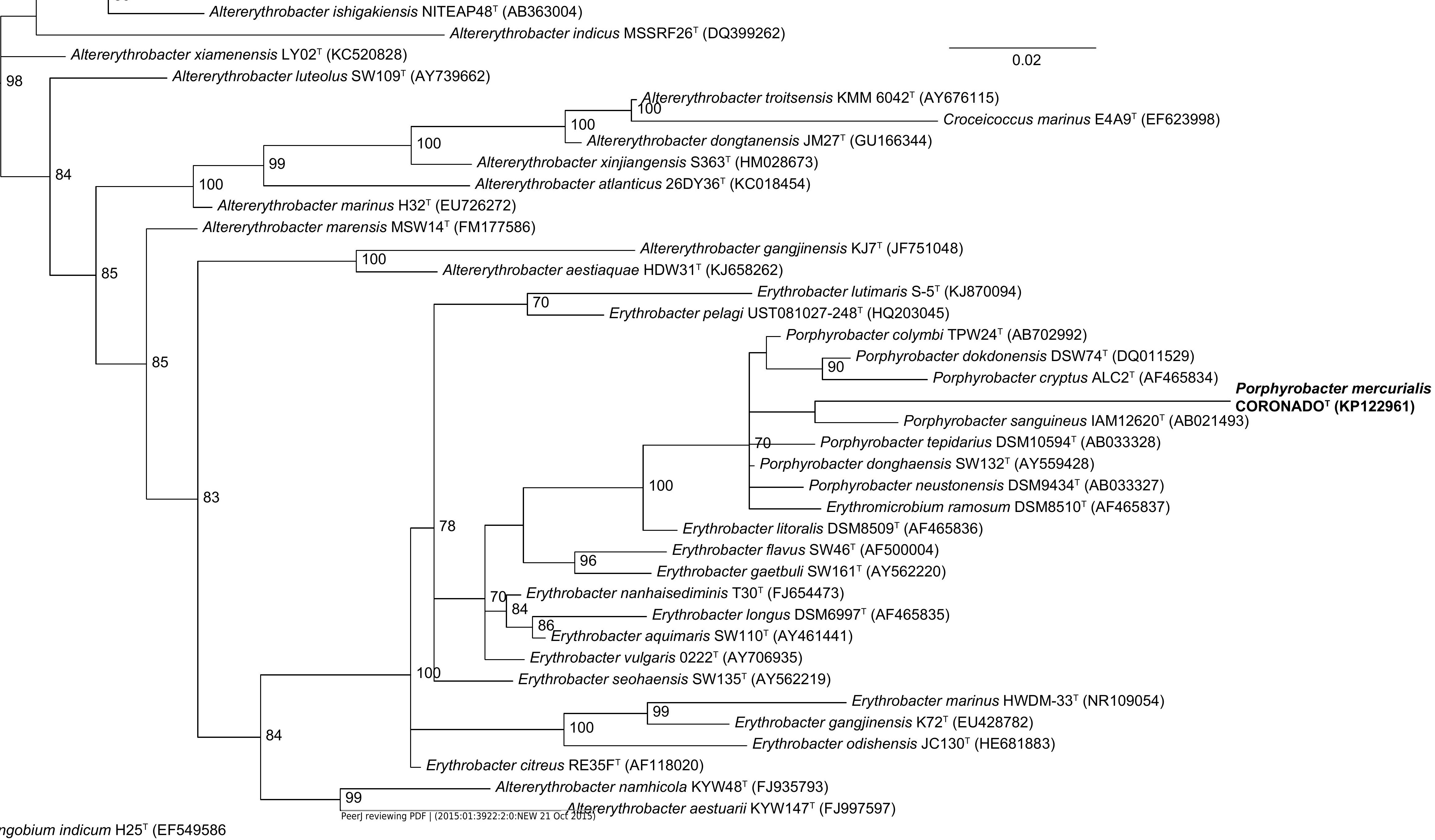


4

Figure 4: Two dimensional silica gel thin layer chromatography results for polar lipids, isolated following standard protocols at the DSMZ (see Methods).

$\mathrm{PL}=$ phospholipid, $\mathrm{PG}=$ phosphatidylglycerol, $\mathrm{PC}=$ phosphatidylcholine, $\mathrm{PE}=$ phosphatidylethanolamine, $\mathrm{DPG}=$ diphosphatidylglycerol, $\mathrm{SGL}=$ Sphingoglycolipid .

\section{PUDPG}

\section{PE}

SGL

PG

PL 


\section{Table $\mathbf{1}$ (on next page)}

Phenotypic comparison of Coronado ${ }^{\top}$ and other members of the Porphyrobacter genus

${ }^{*} \mathrm{C}$, Cocci; O, oval; R, rod. $\mathrm{Y}$, Yellow; O, orange; R, red. Positive $=+$, negative $=-$, No data available=ND. Data from this study and (Hiraishi 2002a) , (Rainey 2003) , (Hanada 1997) , (Yoon 2004), (Fuerst 1993) , (Yoon 2006) . 


\begin{tabular}{|c|c|c|c|c|c|c|}
\hline Characteristic & $\begin{array}{c}\text { strain } \\
\text { CoronadoT }\end{array}$ & $\begin{array}{c}P . \\
\text { sanguineu } \\
S \\
\end{array}$ & $\begin{array}{c}P . \\
\text { tepidariu } \\
S \\
\end{array}$ & $\begin{array}{c}P . \\
\text { donghaensis }\end{array}$ & $\begin{array}{c}P . \\
\text { cryptus }\end{array}$ & $\begin{array}{c}P . \\
\text { neustonensi } \\
S \\
\end{array}$ \\
\hline Cell shape & $\mathrm{O} / \mathrm{R}$ & $\mathrm{R}$ & $\mathrm{O} / \mathrm{R}$ & $\mathrm{C} / \mathrm{O} / \mathrm{R}$ & $\mathrm{R}$ & $\mathrm{C} / \mathrm{O} / \mathrm{R}$ \\
\hline Color of colony & YO & OR & 0 & OR & OR & OR \\
\hline Motility & - & + & - & - & + & + \\
\hline $\begin{array}{l}\text { Presence of } \mathrm{BChl} \\
a \\
\text { Growth in } \mathrm{NaCl} \\
(\%):\end{array}$ & - & + & + & + & + & + \\
\hline Range & $.1-1.5$ & ND & $0.0-1.3$ & ND & ND & ND \\
\hline Optimum & .5 & 1 & ND & 2 & ND & ND \\
\hline Growth $\mathrm{pH}$ : & & & & & & \\
\hline Range & $6.0-8.0$ & ND & ND & ND & $6.0-9.0$ & ND \\
\hline $\begin{array}{l}\text { Optimum } \\
\text { Growth } \\
\text { temperature }\left({ }^{\circ} \mathrm{C}\right)\end{array}$ & 7.0 & 7.0-7.5 & ND & $7.0-8.0$ & $7.5-8.0$ & ND \\
\hline Range & $4-28$ & $20-37$ & ND & $10-45$ & ND & $10-37$ \\
\hline Optimum & 25 & 30 & $40-48$ & $30-37$ & 50 & ND \\
\hline Catalase & + & + & ND & + & + & + \\
\hline Oxidase & - & + & - & + & + & - \\
\hline $\begin{array}{l}\text { Major cellular } \\
\text { fatty acids }\end{array}$ & $\begin{array}{c}C_{18: 1} \omega 7 \text { cis, } \\
C_{16}\end{array}$ & $\mathrm{C}_{18: 1} \omega 7 \mathrm{cis}$ & ND & $\begin{array}{l}C_{18: 1} \omega 7 \text { cis, } \\
C_{17: 1} \omega 6 \text { cis }\end{array}$ & $\begin{array}{c}C_{18: 1} \omega 7 c i \\
s\end{array}$ & n18:1 \\
\hline $\begin{array}{l}\text { DNA G + C } \\
\text { content }\end{array}$ & 67.3 & $\begin{array}{l}63.8-64 \\
\text { (mol\%) } \\
\end{array}$ & $\begin{array}{c}65 \\
(\mathrm{~mol} \%) \\
\end{array}$ & $\begin{array}{c}65.9-66.8 \\
\text { (mol\%) }\end{array}$ & $\begin{array}{c}66.2 \\
\text { (mol\%) }\end{array}$ & $\begin{array}{c}\text { 65.7-66.4 } \\
\text { (mol\%) }\end{array}$ \\
\hline
\end{tabular}

$2{ }^{*}$ C, Cocci; O, oval; R, rod.

$3+Y$, Yellow; O, orange; $R$, red

4 Phenotypic comparison of Coronado ${ }^{\top}$ and other members of the Porphyrobacter genus. Positive $=+$,

5 negative=-, No data available=ND. Data from this study and (Hiraishi 2002a), (Rainey 2003), (Hanada

6 1997), (Yoon 2004), (Fuerst 1993), (Yoon 2006). 